\title{
Radiofrequency ablation of airway smooth muscle for sustained treatment of asthma: preliminary investigations
}

\author{
P.G. Cox*, J. Miller", W. Mitzner", A.R. Leff ${ }^{+}$
}

Radiofrequency ablation of airway smooth muscle for sustained treatment of asthma: preliminary investigations. P.G. Cox, J. Miller, W. Mitzner, A.R. Leff. (C) ERS Journals Ltd 2004.

ABSTRACT: Bronchial thermoplasty is a procedure now being tested in humans for the treatment of asthma. Current studies focusing on safety are encouraging. The procedure, which causes extensive ablation of airway smooth muscle (ASM), is well tolerated, and there is a sustained reduction in airway responsiveness to methacholine. Two assumptions underlie the development of this procedure: 1) ASM is a vestigial tissue; and 2) that treatment directed at ASM alone will provide sustained symptomatic and physiological improvement in asthmatic humans. Even if this procedure is efficacious, it must be safe in the long-term. Current studies in animals and humans suggest that this is very likely to be the case.

While bronchial thermoplasty may have a broad application, especially for patients who wish for a permanent amelioration of their symptoms or have difficulty adhering to medical regimens, the compelling use of this procedure is for patients who are inadequately controlled on current drug therapy or who cannot adhere to therapeutic regimens. The application of this procedure for the treatment of asthma is currently being considered by regulatory agencies, and study centres are currently disseminated throughout North America and Europe. Within the next 1-2 yrs, a profile of the potential role of this therapy in human asthma should be developed fully. Eur Respir J 2004; 24: 659-663.
*Dept of Medicine and \# Surgery, St. Joseph's Healthcare, McMaster University, Hamilton, Ontario, Canada. "School of Public Health, Johns Hopkins Medical Institutions, Baltimore, MD, and ${ }^{+}$Dept of Medicine, Neurobiology, Pharmacology and Physiology and Committee on Clinical Pharmacology and Pharmacogenomics, University of Chicago, IL, USA.

Correspondence: A.R. Leff, Dept of Medicine, MC6076, The University of Chicago Hospital, 5841 South Maryland Ave, Chicago, IL 60637 , USA.

Fax: 17737021859

E-mail: aleff@medicine.bsd.uchicago.edu

Keywords: Asthma airway smooth muscle, asthma treatment

Received: May 62004

Accepted after revision: July 72004

A. Leff is a retained consultant to Asthmatx. Within the past 5 yrs, G. Cox and J. Miller have received honoraria and/or research support for consultations or experimental protocols with Asthmatx, Inc. No part of this paper was written or reviewed by Asthmatx personnel before submission.
ASM contraction is an invariable cause of bronchoconstriction, and relaxation of the airway contractile response with $\beta_{2}$-adrenoceptor bronchodilating agents is a first step in the treatment of symptomatic asthma [1]. In the past decade, the efficacy of combined anti-inflammatory agents and longacting $\beta_{2}$-adrenoceptor agonists has made them the mainstay of therapy in patients with moderate and severe asthma [2]. For many patients, the combined use of anti-inflammatory agents with inhaled bronchodilators produces excellent longterm control of asthma. Yet, serious exacerbations still recur, even in patients with mild asthma [3], and some patients with severe asthma are poorly responsive to all forms of therapy, including high-dose oral corticosteroids. The mechanism by which airway inflammation is translated into airway narrowing remains to be elucidated, and there is currently a poor understanding of the mechanisms for the global lack of pharmacological responsiveness in patients with moderate or severe refractory asthma. It has been hypothesised that hyporesponsiveness to drug therapy in asthma is, in part, genetically determined, and preliminary data suggest that $\beta$ adrenoreceptor polymorphisms may, in part, contribute to this problem in some patients [4].

The concept of treating airways with heat generated by radiofrequency energy is based upon the supposition that blockade of the bronchial smooth muscle tone in asthmatic patients not responding optimally to conventional therapy could cause amelioration of chronic symptoms and reduce exacerbations. This concept assumes that blockade of ASM responsiveness in the conducting airways of the lung will attenuate the bronchoconstrictor response in chronic asthma that is not satisfactorily controlled with pharmacotherapy. A second target group might be patients with recurrent asthma who are unable to adhere to therapeutic regimens over a lifetime. This review examines pre-clinical evidence in animals (dogs) and some early clinical data, which define the ability of local application of heat to the airways in attenuating the bronchoconstrictor response to methacholine. Because most of these data are published only in abstract form, those findings must be considered preliminary data, and final outcomes cannot be established at this time.

Two major concerns underlie the application of radiofrequency therapy (RFT) to airways with the intent to reduce the potential of the smooth muscle to contract. First, ASM must serve no physiologically essential role in human airways. Humans and, rarely, cats are the only species that develop true asthma, and the details of the vestigial function of ASM are defined in the first part of this perspective.

The second concern is that the RFT that is efficacious is also safe. A premise of RFT is that ASM will either be 
inactivated or obliterated without any long-term alteration of other tissues and that airway function will remain normal (with reduced bronchoconstriction) for the lifetime of the patient. Preliminary data in dogs and humans have been presented from early stage investigations, and these are next presented in this perspective.

\section{Role of smooth muscle in asthma}

Asthma is an often debilitating disease characterised by dyspnoea, wheezing, coughing, respiratory distress, and sometimes death. Subjects with asthma typically have hyperresponsive and, often, chronically inflamed airways. Chronic asthma is also characterised by extensive airway remodelling [5], with a thickening of airway walls, increased mucus glands and goblet cells, increased vascularisation, and most importantly, hypertrophy of ASM. Although there are many different triggers, an acute asthma attack is always characterised by contraction of the smooth muscle in the airway wall. Much recent research in asthma has focused on developing an understanding of the factors that mediate the tissue response in exacerbations of asthma [6]. Indeed, several new treatments for the disease involve blockade or inhibition of mediators involved in these pathways [7, 8], or prevention of activation of pathways initiated by blockade of antigen cross-linking on mast cells [9]. While it is true that increasing knowledge of immunology pathways has a therapeutic value in asthma, the fact remains that an asthma attack can often be triggered by nonallergic stimuli, such as infection, exercise or cold air. Nevertheless, whether the initial cause results from an allergen, an irritant, infection, psychological stress or other neural activation, the cascade always leads to airway muscle contraction. It would, thus, seem most sensible to treat asthma by minimising the ability of this smooth muscle to contract. Despite considerable research concerned with ASM function and regulation, there has not been strategies developed that would alter the muscle such that its ability to shorten in vivo is chronically impaired.

\section{Which airways cause the problem in asthma?}

One important question is which level of airways contributes most to airflow obstruction during an asthma attack. This is a critical issue that bears on the whole approach to using RFT, since this technique clearly cannot treat the more distal, membranous airways. However, after many years of debate on whether large or small airways are more responsible for airflow obstruction during asthma attacks, there remains little experimental evidence on which to base any conclusive answer. It is well accepted and known from morphometric models of Weibel [10], Horsfield and Cumming [11, 12], and others [13], that nearly all of the baseline airway resistance lies in the conducting airways $>2 \mathrm{~mm}$. With smooth muscle contraction, this partitioning can surely change, but this situation has not been extensively modelled. Indeed, for many years it was thought that the structure of large airways was such that they could not constrict as much as the smaller ones. However, recent work [14] showing that even large cartilaginous airways could narrow to complete closure if sufficiently stimulated, dispelled that myth. PELlegrino et al. [15] have demonstrated that complete airway closure could possibly also be achieved in humans. However, even if this is the case in humans, the locus of airway constriction during an acute asthma attack is not fully elucidated. Is it a global narrowing of small airways, an acute narrowing of a few large airways, or a generalised narrowing of all airways in the entire bronchial tree? Lacking any convincing experimental evidence to the contrary, it can be assumed that it is the simplest explanation, which is that it is a generalised narrowing of all airways. Since, as mentioned, most of the airway resistance at baseline lies in the larger airways, an equivalent degree of narrowing throughout the airway tree will have its greatest impact on resistance in these larger airways. For this reason, it is reasonable to think that impairment of the ability of the conducting airways to narrow could have a substantially beneficial effect on the ability to breathe during an asthma attack.

\section{What is the function of airway smooth muscle?}

The human body contains many organs, which no longer provide evolutionary advantage and have no known function, e.g. the appendix, most body hair, wisdom teeth, male nipples, and external ear muscles. It recently has been argued [16] that ASM falls into this category: that of an organ with no known physiological purpose, whose sole contemporary contribution is the potential to cause problems. This argument supports an earlier discussion by SEOw and FREDBERG [17], who emphasised that there is no known disease entity or physiological deficit associated with loss of ASM. They further suggested that airway smooth muscle was perhaps a vestigial remnant of its common embryological origin with the gastro-intestinal system. So from where does the widespread feeling that smooth muscle must have some function arise? Some of this background is summarised below.

Despite the fact that the function of smooth muscle in the airway wall has been speculated for many years, from a functional perspective, there has never been strong experimental evidence for its presence in the lung. Notwithstanding this lack of experimental evidence, MACKLIN [18], in his extensive and influential review article on ASM, could not imagine that such a system existed for no physiological purpose, stating, "Organized as it is, into a very complex system, this muscle would seem of the utmost functional importance, in fact quite indispensable in respiration."

At least 10 postulated roles for ASM have appeared in the literature. These possible roles consist of the following: 1) peristalsis to assist exhalation; 2) peristalsis to assist mucus propulsion; 3) peristaltic contraction in the foetal lung to generate fluid pressure; 4) promoting lymphatic and venous flow; 5) ventilation/perfusion matching; 6) protecting the peripheral lung; 7) protecting airway structure; 8) stabilising airways; 9) enhancing the effectiveness of cough; and 10) optimising anatomic dead space volume. Details of each of these have been presented elsewhere $[16,18]$ and will not be repeated here. However, it should be noted that none of these potential functions of ASM have been shown to be essential to normal lung physiology. If ASM were eliminated, then the airways might enlarge slightly, but there would be no other obvious physiological consequence. Thus, the evidence strongly supports the suggestion that ASM is indeed a vestigial organ [17], quite analogous to the appendix, which also has no known purpose other than to cause serious medical problems. Such being the case, if there were a way to treat ASM like an inflamed appendix, that is, to effectively cut it out, then asthma, like appendicitis, could be cured. Airway inflammation with associated secretions may still be present, but without an ability to translate inflammatory signals into ASM contraction, dyspnoea and life-threatening hypoventilation from airway closure and respiratory muscle fatigue would be greatly minimised. 


\section{Pre-clinical studies using radiofrequency thermal therapy in canine airways}

Most available data for efficacy and histology in RFT come from works still in progress; one peer-reviewed publication is in press [19]. All other data cited in this section refer to data from dogs, which have been presented only in abstract form. Preliminary studies in dogs suggest that radiofrequency thermal treatment can reach airways $>3 \mathrm{~mm}$ in diameter [19]. Airways are reached through a bronchoscope, and an expandable basket with four electrode arms is opened to make contact with the airway wall circumferentially (fig. 1). Initial treatment generally causes blanching at the site of treatment, and histology shows epithelial disruption at treated sites. As such, this treatment is not a burn comparable to cardiac electrophysiological ablation or in the sense of cauterisation. There is no charring of the treated area, and subsequent epithelial re-growth is complete. The only residual disruption of morphology is the replacement of ASM at the treatment site by loose connective tissue. All other tissue elements remain intact or return to normal as assessed by full thickness histological sections taken at sacrifice at various time intervals up to 3 yrs later.

Preliminary studies have been performed in dogs to determine the optimal temperature and treatment times [19] to effect selective ablation of ASM without causing long-term damage to other tissues. Smooth muscle appears to be uniquely heat sensitive, which allows for the selective effect of RFT. Initial studies mapped different sites in the airways of

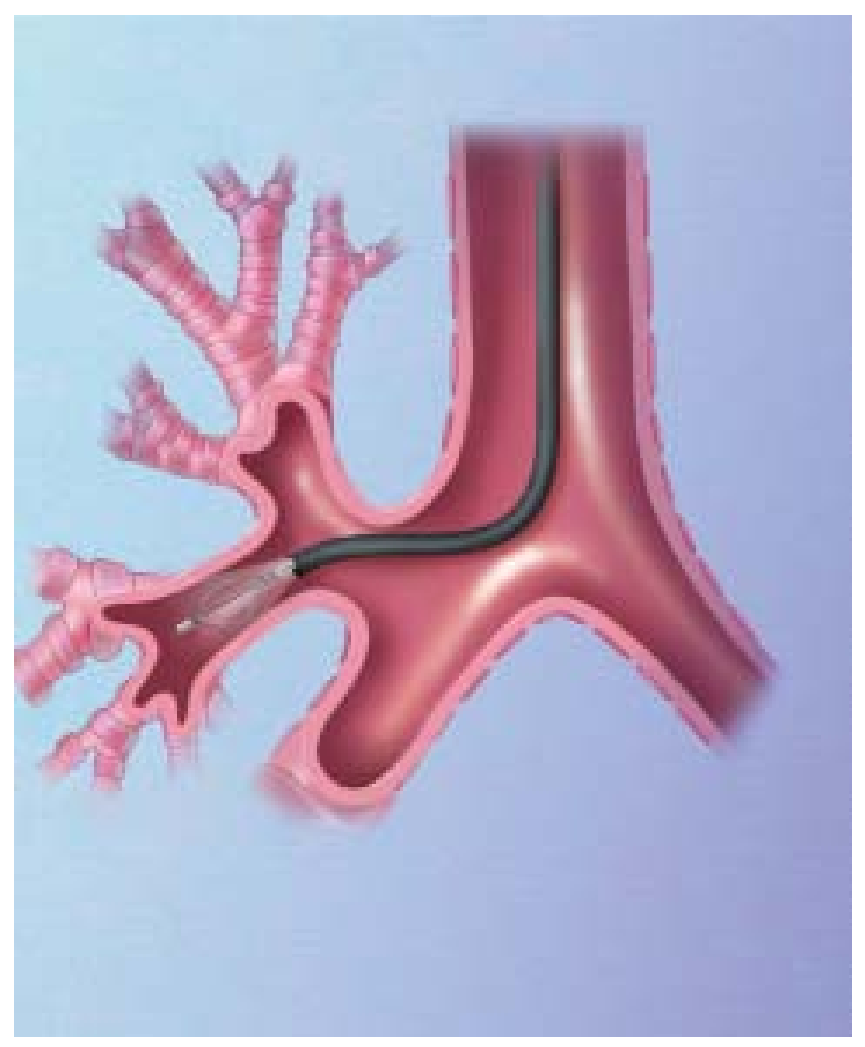

Fig. 1.-Schematic illustration of the instrument used to perform radiofrequency alteration of airway smooth muscle responsiveness in dogs and humans. The catheter conducts radiofrequency energy to the airway by direct contact to heat the airway wall. The bronchoscope is direct to the area, the basket then is expanded, radiofrequency heat treatment is activated for a very short duration, the basket is withdrawn and the instrument is directed to the next site

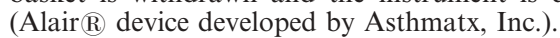

the same dogs so that these sites could be revisited for visual and, eventually, histological examination. The major variables at each site were temperature and duration of treatment. Because multiple sites at each setting were examined, the optimal settings could be determined within the same animal. Airway calibre was measured optically [20]; these data have provided treatment parameters in dogs, which result in substantial and prolonged attenuation of airway constriction to locally applied methacholine (fig. 2). This effect persisted for 3 yrs. As noted above, histological changes are selective for ASM. There is no stricture of airways, generalised fibrosis beyond the boundaries of the ASM, or retained mucus in the airways. The degree of attenuation of the contractile response to methacholine appears to correlate well to the degree of ASM loss [19].

Using parameters determined from studies of temperature and duration of RFT in dogs, the feasibility of treating conducting airways of the human lung (see next section) has been examined.

After treatment, some degree of airway responsiveness to locally applied methacholine still remains in airways in dogs in which smooth muscle appears to be replaced by loose connective tissue. Two possibilities for the residual degree of airway responsiveness are suggested. As the effect is localised to the site of treatment, smooth muscle between treatment sites may be less affected; this muscle being sufficiently contractile to cause some airway narrowing upon challenge. A second consideration is that the treatment process is designed to be minimally destructive, rather than ablative in the sense of cauterisation. Hence, the muscle at some treatment sites might not be fully ablated by RFT, which is designed to err on the side of safety for human application (see below).

\section{Studies of radiofrequency thermal therapy in human asthmatic subjects}

With the experience gained from the extensive studies in animals the technology for the application of heat to human airways was developed. In humans, this therapeutic application is termed "bronchial thermoplasty". The first studies were carried out in patients who were scheduled to undergo pulmonary resection, typically lobectomy for suspected neoplasm [22]. Those subjects scheduled to have a bronchoscopy for preoperative evaluation 1-3 weeks prior to surgery were enrolled. This provided the opportunity to carry out the thermoplasty procedure in airways that would be available for histological examination soon thereafter. While this did not afford the opportunity to examine a functional outcome or the long-term results, it did provide an ideal opportunity to evaluate whether the human airway responded acutely in a similar fashion to the canine airway for which there is extensive prior experience [19]. Specifically, this study design enabled the evaluation of changes in the smooth muscle mass in the human airway. As bronchoscopy was repeated at the time of surgery, there was also opportunity to inspect the bronchial lining at the sites of treatment to evaluate local consequences.

The eight subjects treated in this study had no adverse events related to the procedure, and there were no interruptions or delays to the planned management of their primary clinical problem. Histological examination of the airways indicated a remarkably similar response in human and canine airways [23]. The effect of treatment localised primarily to the airway wall and was typically focal in distribution, as would be expected from the design of the treatment device. The expected reduction in mass of smooth muscle in the airway wall occurred with treatment. This experience was 
fundamental in enabling the design of clinical studies in patients with asthma.

Transfer of this technology to use in patients with asthma is a major step that requires prolonged negotiation with regulatory agencies including governmental health agencies and institutional research ethics boards. The concept of causing a physical change in the airway that would be expected to be of long-term, even permanent, duration is both novel and radical, particularly in the context of a disease such as asthma where there has been major development of pharmacological therapies during the last 3 decades. However, despite these advances in drug treatment, there remains a population of patients with significant morbidity from their asthma. These patients may have resistance to the expected effects of drug treatment, reduced access to or compliance with prescribed medication, or asthma with the potential to develop substantial bronchoconstriction with little prodrome or warning. With the information gained from large groups of patients studied over longer periods of time, we now know that serious exacerbations occur even in patients with mild asthma. Such exacerbations can occur despite excellent therapy, indicating an opportunity for development of a complementary approach to drugs for optimally treating asthma. However, it is not yet known if bronchial thermoplasty will cause an overall reduction in the level of asthma severity.

A second concern is the potential for damage to the airway to develop over time, perhaps as a result of progressive injury and fibrosis. This adverse outcome has not been observed over 3 yrs of follow-up in animals. The 16 subjects with mild or moderate asthma have been followed for $>12$ months without any evidence of chronic or progressive airway injury [21]. The histological studies in animals showed no evidence of chronic inflammation; in fact, there was prompt resolution of the response to treatment so that there were no inflammatory cells evident beyond 12 weeks after treatment [23]. While this concern can only be completely dismissed by extensive clinical experience, the data obtained to date are reassuring, and it seems unlikely that progressive airway narrowing would occur in the absence of a persisting or recurring stimulus.

The first study of the procedure in patients with asthma was designed with the primary focus on safety [24]. Thus, the major objective of the initial study was to determine whether it was feasible to carry out bronchial thermoplasty in patients with asthma; for this it would be necessary to establish that subjects would volunteer for this study, that it could be accomplished safely, and that there would be appropriate short and long-term evaluations. To perform the procedure in the safest manner, it was agreed initially that it would be performed under general anaesthetic in the operating room with placement of an endotrachial tube. This would allow for maximum control of the airway. Once it was established that the procedure could be performed as expected and completed in $30 \mathrm{~min}$ with modest post-procedure increase in airway symptoms, a major change to carrying out the procedure under local anaesthetic was made. There are no systematic differences in the adverse events attributable to the procedure when performed either under local or general anaesthetic. There are distinct patient preferences for one or the other. Obviously it is logistically simpler to carry out the procedure under local anaesthetic if the treatment team is comfortable carrying out a procedure of $30 \mathrm{~min}$ duration.

At the time of treatment, there is usually little if any direct evidence of heat effect at a treatment site. At times a faint pale stripe is visible. More commonly, however, there is evidence of acute epithelial desquamation and mucous secretion. This latter phenomenon has led to the routine use of drying agents during the procedure. Despite the routine use of pre-operative

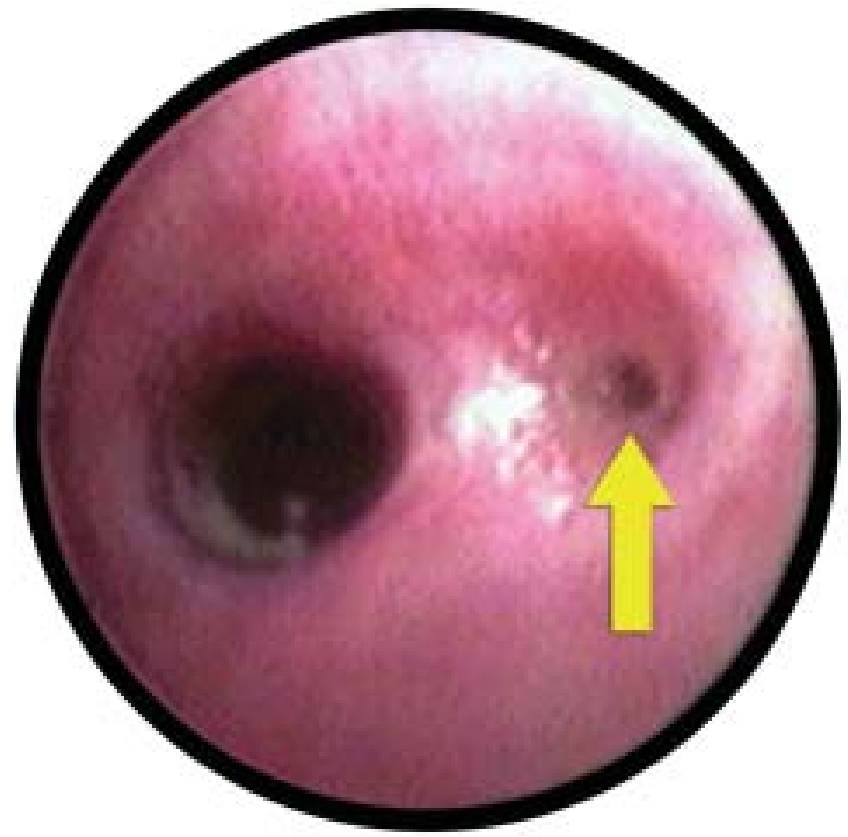

Fig. 2.-Effect of radiofrequency heat treatment to the adjacent branching canine airways. The airway on the left received treatment and did not constrict by local administration through the bronchoscope of methacholine. By contrast, the untreated airway constricts nearly to closure with the same stimulus. Persistence of effect is sustained [21].

bronchodilator therapy it is not uncommon to see transient local bronchoconstriction. On rare occasions and particularly when deploying the catheter in smaller airways, minimal bleeding is encountered. This has been a self-limited process.

At present, the procedure is carried out only in those airways that are accessible at bronchoscopy and can be treated under direct vision. This has been the practice so as to enable subsequent examination of all treated sites. With the confidence provided by greater experience, it may be appropriate also to treat airways of sufficient size that are beyond the field of direct vision. The right middle lobe is not treated, because of concern that the long and narrow airway that typically leads to the right middle lobe may make this area more prone to chronic damage. The consideration that the ventilatory capacity of the middle lobe is modest led to the current approach of avoiding treatment in this area.

In the days after treatment there is an increase in the frequency of airway symptoms, such as cough, mucous production, hoarseness and dyspnoea [24]. Examination of daily records of peak flows shows modest reduction suggesting that the symptoms are primarily those of bronchial irritation rather than airflow obstruction. The symptoms experienced by subjects after bronchial thermoplasty are similar to those experienced by other subjects with asthma undergoing bronchoscopy and related procedures. While there has been frequently an increase in symptoms that are attributable to the procedure, none of these have been severe or serious. Most of these symptoms have improved spontaneously or as a result of using commonly available medications as needed.

The successful experience, which showed that radiofrequency therapy could be performed in patients with asthma under either local or general anaesthetic, enabled the design of a clinical trial examining the effectiveness of this procedure in subjects with moderately severe asthma [25]. This trial is currently underway at a number of sites in Europe and North America. One intriguing result of the feasibility study was 
the finding of a significant improvement in bronchial responsiveness as assessed by methacholine challenge up to 12 months later [21]. It will be of interest to examine whether this improvement in bronchial responsiveness persists and whether it is associated with improvement in clinical control of asthma.

\section{References}

1. Murray PT, Corbridge T. Pharmacotherpy of Asthma. In: Hall JB, Corbridge TC, Rodrigo C, Rodrigo G, eds. Acute Asthma: Assessment and Management. New York, McGraw-Hill Companies Inc., 2000; pp. 139-160.

2. National Institutes of Health, National Heart and Lung Institute. Practical Guide for the Diagnosis and Management of Asthma. US Department of Health and Human Services, NIH Publication No. 97-4053, October, 1997.

3. O'Byrne P, Barnes PJ, Rodriguez-Roisin R, et al. Low dose inhaled budesonide and formoterol in mild persistent asthma. Am J Respir Crit Care Med 2001; 164: 1392-1397.

4. Israel E, Drazen JM, Liggett SB, et al. The effect of polymophisms on the $\beta_{2}$-adrenergic receptor on the response to regular use of albuterol in asthma. Am J Respir Crit Care Med 2000; 162: 75-80.

5. Carroll N, Elliot J, Morton A, James A. The structure of large and small airways in nonfatal and fatal asthma. Am Rev of Respir Dis 1993; 147: 405-410.

6. Nadel JA, Busse WW. Asthma. Am J Respir Crit Care Med 1998; 157: S130-S138.

7. Drazen JM. Asthma therapy with agents preventing leukotriene synthesis or action. Proc Assoc Am Physicians 1999; 111: 547-559.

8. Leckie $\mathrm{MN}$, ten Brinke A, Knan J, et al. Effect of an interleukin-5 blocking monoclonal antibody on eosinophils, airway hyperesponiveness and the late asthmatic response. Lancet 2000; 356: 2144-2148.

9. Busse W, Corren J, Lanier BQ, et al. Omalizumab, anti-IgE recombinant humanized monoclonal antibody, and the treatment of severe allergic asthma. J Allergy Clin Immunol 2001; 108: 184-190.

10. Weibel ER. Morphometry of the Human Lung. New York: Academic Press, 1963.
11. Horsfield K, Cumming G. Morphology of the bronchial tree in man. J Appl Physiol 1968; 24: 373-383.

12. Horsfield K, Cumming G. Morphology of the bronchial tree in the dog. Respir Physiol 1976; 26: 173-182.

13. Pedley TJ, Schroter RC, Sudlow MF. Gas flow and mixing in the airways. In: West JB, ed. Bioengineering Aspects of the Lung. New York: Marcel Dekker Inc., 1977, pp. 163-265.

14. Brown RH, Mitzner W. The myth of maximal airway responsiveness in vivo. J Appl Physiol 1998; 85: 2012-2017.

15. Pellegrino R, Biggi A, Papaleo A, Gianfranco C, Rodarte JR, Brusasco V. Regional expiratory flow limitation studied with Technegas in asthma. J Appl Physiol 2001; 91: 21902198.

16. Mitzner W. Airway smooth muscle: the appendix of the lung. Am J Respir Crit Care Med 2004; 169: 1-4.

17. Seow CY, Fredberg JJ. Historical perspective on airway smooth muscle: the saga of a frustrated cell. J Appl Physiol 2001; 91: 938-952.

18. Macklin CC. The musculature of the bronchi and lungs. Physiol Reviews 1929; 9: 1-60.

19. Danek CJ, Lombard CM, Dungworth DL, et al. Reduction in airway hyperresponsiveness to methacholine by the application of RF energy in dogs. J Appl Physiol 2004; (In press).

20. Miller P, Danek CJ, Cox G, Miller J. Development of a bronchoscopic technique for airway diameter measurement. Chest 2001; 120: 229s

21. Cox G, Miller J, McWilliams A, Fitzgerald M, Lam S. Bronchial thermoplasty: one-year update. Am J Respir Crit Care Med 2004; 169: A313.

22. Miller JD, Cox G, Vincic L, Lombard CM, Loomas BE, Danek CJ. Bronchial thermoplasty is well tolerated by nonasthmatic patients requiring lobectomy. Am J Respir Crit Care Med 2002; 165: A216.

23. Lombard CM, Vincic L, Cox G, et al. Histological effects of bronchial thermoplasty of canine and human airways. Am J Respir Crit Care Med 2002; 165: A779.

24. Cox G, Miller JD, Noth I, et al. Early clinical experience with bronchial thermoplasty for the treatment of asthma. Am $J$ Respir Crit Care Med 2002; 165: A187.

25. Laviolette $\mathrm{M}$, Thomson $\mathrm{N}$, Niven $\mathrm{R}$, et al. Asthma intervention research (AIR) trial: early safety assessment of bronchial thermoplasty. Am J Respir Crit Care Med 2004; 169: A314. 